\title{
Susceptibility to sensitization. I. Sex differences in the enduring effects of chronic D-amphetamine treatment on locomotion, stereotyped behavior and brain monoamines
}

\author{
Dianne M. Camp ${ }^{1}$ and Terry E. Robinson ${ }^{1,2}$ \\ ${ }^{1}$ Department of Psychology and ${ }^{2}$ Neuroscience Program, The University of Michigan, Ann Arbor, MI 48109 (U.S.A.)
}

(Received 3 November 1987)

(Revised version received 1 February 1988)

(Accepted 5 February 1988)

Key words: Sensitization; Amphetamine; Sex difference; Dopamine; Striatum; Stereotyped behavior; Locomotion; Chronic drug treatment

\begin{abstract}
There are sex differences in a number of behaviors elicited by amphetamine (AMPH). The purpose of the present experiment was to determine if there are also sex differences in the sensitization of the locomotor activity and stereotypy produced by repeated intermittent AMPH treatment, and whether this is accompanied by sex differences in dopamine (DA) metabolism. It was found that female rats showed greater and more rapid sensitization of locomotor activity and stereotyped behavior than males. In addition, prior exposure to AMPH was associated with an elevation in resting striatal dihydroxyphenylacetic acid (DOPAC) to DA ratios in female, but not male rats, suggesting a sex difference in one neurochemical correlate of sensitization. As a group, males were more variable and heterogeneous in their response to repeated AMPH treatment, because they were divisible into two neurochemically distinct subgroups on the basis of their change in behavior and females were not. This heterogeneity may make it more difficult to identify neurochemical correlates of sensitization in males. It is suggested that there is a sex difference in the responsiveness of brain DA systems to repetitive activation, and this contributes to individual variation in the susceptibility to sensitization.
\end{abstract}

\section{INTRODUCTION}

The repeated intermittent administration of psychomotor stimulant drugs produces a progressive enhancement in many stimulant-induced behaviors, a phenomenon known as behavioral sensitization. For example, the locomotion, stereotypy and rotational behavior produced by amphetamine (AMPH) usually show a more rapid onset, an increased intensity, or a longer duration with successive injections ${ }^{12,24,33,44,61}$. Although most animals are sensitized by repeated AMPH administration, there are also large individual differ- ences in the rate and extent of sensitization $^{30,36,42,45,60}$. Little is known, however, about factors which influence the development of sensitization. Genetic factors clearly contribute to individual variation in the susceptibility to sensitization, but how they do so is unknown ${ }^{15,30,45,58}$. In addition, the sex of an animal and the presence of testicular hormones modulate the sensitization of AMPH-induced rotational behavior ${ }^{4,50}$. Female rats show greater sensitization of AMPHelicited rotational behavior than males, and castration of male rats enhances the sensitization of

Correspondence: T.E. Robinson, Neuroscience Laboratory Building, The University of Michigan, 1103 E. Huron St., Ann Arbor, MI 48109, U.S.A. 
rotational behavior. These latter studies suggest that a testicular hormone directly or indirectly retards the development of sensitization.

It is not known, however, if there are sex differences in the sensitization of other AMPHinduced behaviors, such as locomotion or stereotypy. AMPH produces many different behavioral effects by altering activity in multiple and functionally heterogeneous mesotelencephalic dopamine (DA) systems (refs. 11,19,23,65 for reviews). It is possible, therefore, that the sex of an animal influences the sensitization of other AMPH-induced behaviors differently than rotational behavior. To explore this issue the present experiment was designed to determine the influence of sex on the sensitization of locomotion and stereotyped behavior produced by repeated AMPH treatment, and on associated changes in DA metabolism $^{48,49}$.

\section{MATERIALS AND METHODS}

\section{Subjects}

Adult male and female Sprague-Dawleyderived Holtzman rats (Holtzman Co., Madison, WI) initially weighing $250-350 \mathrm{~g}$ were housed individually in a light-controlled room $(14: 10 \mathrm{~h}$ light : dark cycle, lights on at $08.00 \mathrm{~h}$ ) and provided with free access to food and water. All treatment and test procedures were carried out between 08.00 and $17.00 \mathrm{~h}$.

\section{Apparatus for recording motor activity}

Continuous measures of locomotor activity were obtained with Digiscan Animal Activity Monitors (Omnitech Electronics, Columbus, $\mathrm{OH}$ ), which detect movement by use of 32 infrared light beam sensors and emitters located $2.54 \mathrm{~cm}$ apart about the perimeter of a Plexiglas test chamber $(40.6 \times 40.6 \times 30.5 \mathrm{~cm})$. The monitors were connected to a microprocessor (Digiscan Analyzer, Omnitech Electronics) that automatically recorded several different measures of motor activity over 5-min intervals. The data from the Digiscan system presented here represent the distance animals traveled in inches, which was estimated by interpolating the distance between successive beam interruptions.

\section{Rating stereotyped behavior}

In addition to the automated analysis of locomotion described above, the animals were rated for stereotyped behavior during $1 \mathrm{~min}$ observation periods at 10 and $20 \mathrm{~min}$ following the injection, and every $20 \mathrm{~min}$ thereafter for a total of $2 \mathrm{~h}$ (i.e. a total of 7 ratings). The rater was blind to the treatment conditions. Two different rating scales were used. (a) An overall stereotypy score was based on the scale described by Ellinwood and Balster ${ }^{13}$. On this scale, $1=$ asleep (lying down, eyes closed), 2 = inactive (lying down, eyes open), 3 = normal in place activities (normal grooming, occasional walking, turning), 4 = normal, alert, active (moving about cage, sniffing or rearing intermittently), 5 = hyperactive (running movement characterized by rapid changes in position), $6=$ slow patterned activity (repetitive exploration of cage at normal levels of activity), 7 = fast patterned activity (repetitive exploration of cage with hyperactivity), $8=$ restricted (remaining in the same place with fast repetitive head and limb movements), and $9=$ dyskineticreactive (backing-up, jumping, seizures, abnormally maintained postures, and/or dyskinetic movements). (b) Stereotyped sniffing behavior was rated separately, according to both its duration ( $1=$ discontinuous, $2=$ continuous) and intensity ( $1=$ mild, $2=$ moderate, $3=$ intense $)$, as described by Rebec and $\mathrm{Segal}^{43}$. A single stereotypy score for sniffing at each rating interval was derived by multiplying the duration and intensity scores.

\section{Experimental design and protocol}

Male and female animals received an intraperitoneal (i.p.) injection of D-amphetamine sulfate (AMPH) once every 4 days for a total of 10 injections, but behavior was monitored only following the 1st, 4th, 7th and 10th injections ('test days'). On these test days each animal was removed from its home cage and placed individually in the test chamber. Following a 15-min habituation period, each animal received an injection of AMPH and was immediately replaced in the test chamber for $2 \mathrm{~h}$. On the days when AMPH was administered but behavior was not monitored (i.e. injection days 2, 3, 5, 6, 8 
and 9) the animals were not exposed to the test chambers, but were immediately returned to their home cage following the injection. This was to minimize drug-environment conditioning effects associated with repeated AMPH administration $^{39,41,55,62,64}$ (cf. ref. 59).

Male rats $(n=11)$ always received $3.0 \mathrm{mg} / \mathrm{kg}$ AMPH (weight of the salt) dissolved in $0.9 \%$ saline $(3.0 \mathrm{mg} / \mathrm{ml})$. In contrast, the females $(n=17)$ were subdivided into two groups which received two different doses of AMPH $(2.6 \mathrm{mg} / \mathrm{kg}$ or $1.78 \mathrm{mg} / \mathrm{kg}$ ), for the following reasons. Male and female rats metabolize AMPH at different rates, and females attain slightly higher brain levels of AMPH than males when the same systemic dose is administered ${ }^{3,10,22,34}$. One of the doses $(2.6 \mathrm{mg} / \mathrm{kg})$ controlled for this sex difference in AMPH metabolism by equating brain levels of the drug. In a previous study using rats of the same age, same strain and same supplier, a range (1-10 mg/kg) of systemic doses of AMPH was administered to male and females rats, and whole brain and striatal concentrations of AMPH were measured at various points in time after the injection ${ }^{3}$. From this study it was determined that an i.p. injection of $2.6 \mathrm{mg} / \mathrm{kg}$ to females produced the same brain concentrations of AMPH as did $3.0 \mathrm{mg} / \mathrm{kg}$ in males. Therefore, all females received $2.6 \mathrm{mg} / \mathrm{kg}$ AMPH on the days when AMPH was administered but behavior was not monitored (i.e. injection days 2, 3, 5, 6, 8 and 9). This dose will be referred to as the 'sensitizing' dose. A comparison of males and females receiving equivalent sensitizing doses of AMPH (in terms of brain concentrations) is used as one index of sex differences in the response to repeated AMPH treatment.

A second, and even more conservative control group, was also included. Females in this group $(n=9)$ received $1.78 \mathrm{mg} / \mathrm{kg}$ of AMPH on test days (i.e. days when behavior was monitored). This dose will be referred to as the 'challenge' dose, because on the last injection day (i.e. the day 10 challenge) all the females received the challenge dose of $1.78 \mathrm{mg} / \mathrm{kg}$ (i.e. also females pretreated with $2.6 \mathrm{mg} / \mathrm{kg}$ ). Based on the Becker et al. ${ }^{3}$ study, it is estimated that the challenge dose $(1.78 \mathrm{mg} / \mathrm{kg})$ produced brain levels of AMPH in females that were $33 \%$ lower than in males receiving $3.0 \mathrm{mg} / \mathrm{kg}$ AMPH. For the sake of simplicity, females that received the challenge dose on each test day will be referred to as the 'female$1.78^{\prime}$ group, and females that received $2.6 \mathrm{mg} / \mathrm{kg}$ on all days but the last challenge test day as the 'female-2.6' group.

All animals in each group were also given a single i.p. injection of $0.9 \%$ saline $(1 \mathrm{ml} / \mathrm{kg}) 3-4$ days following the last AMPH injection, and the same procedures were followed as described above (except behavior was recorded for only $1 \mathrm{~h}$ after the injection).

Females were tested without regard for their estrous cycle, and it is recognized that this could increase the variance in this group because of estrous cycle-related fluctuations in dopaminergic activity and response to $\mathrm{AMPH}^{3}$. It was thought, however, that this was preferable to taking daily vaginal smears to track the estrous cycle, which would introduce an additional confounding stressful manipulation; especially since it is known that prior stress interacts with AMPH sensitization $^{1,2,45}$.

\section{Neurochemical methods and procedures}

Eight to 13 days after the last injection of AMPH all animals were killed by decapitation, and the brains were rapidly removed $(<40 \mathrm{~s})$ and placed in ice-cold saline. In addition, a group of naive control male and female rats that received no prior treatment were included. These animals were untreated to exclude the changes in DA activity produced by repeated injections of sa$\operatorname{line}^{51,68}$. The brains were dissected by a person (T.E.R.) blind to treatment conditions. The dissection procedure was very similar to that described by Heffner et al. ${ }^{16}$, except the frontal cortex included only the medial frontal cortex located anterior to the genu of the corpus callosum. The tissue samples were weighed and placed into tubes which contained $250 \mu 1$ of $0.05 \mathrm{~N}$ perchloric acid and $2.0 \mathrm{ng} / 10 \mu \mathrm{l}$ of the internal standard, dihydroxybenzylamine (DHBA). The tissue was homogenized and then centrifuged at $5000 \mathrm{~g}$ for $45 \mathrm{~min}$ at $2-4^{\circ} \mathrm{C}$. The supernatant was filtered using $0.2 \mu \mathrm{m}$ regenerated cellulose membrane filters and stored in vials at $-20^{\circ} \mathrm{C}$. 
The concentrations of dopamine (DA), 3,4dihydroxyphenylacetic acid (DOPAC), 5-hydroxytryptamine (5-HT; serotonin) and 5-hydroxyindoleacetic acid (5-HIAA) were assayed by highperformance liquid chromatography with electrochemical detection (HPLC-EC). Briefly, the HPLC system employed a Brownlee reverse phase $\mathrm{C}-18$ column and an LC-4 electrochemical detector with a glassy carbon electrode (BioAnalytical Systems, Lafayette, IN). The mobile phase was a citric acid-sodium phosphate buffer (pH 3.35) containing $25-30 \mathrm{mg} / 1$ octyl sulfonate and $8-10 \%$ methanol. The detector potential was set at $+0.74 \mathrm{~V}$ versus an $\mathrm{Ag} / \mathrm{AgCl}$ reference electrode.

\section{Statistical analyses}

The stereotypy rating scores were analyzed using non-parametric statistical techniques, including Profile analyses ${ }^{37}$, Friedman's analyses of variance for repeated measures and the Wilcoxon signed-ranks test for matched pairs. Either the median test or the Mann-Whitney $U$-test was used for individual comparisons. (The median test is not as powerful as the Mann-Whitney $U$-test, but is preferable if there are many tied scores $^{35}$.) Locomotor activity scores and the neurochemical data were analyzed using parametric statistics that included one- or two-way analyses of variance for repeated measures, or $t$-tests for individual a priori comparisons. All comparisons were based on two-tailed probabilities, unless otherwise noted.

\section{RESULTS}

\section{Sensitization of stereotyped behavior}

On the challenge test day (day 10) some animals received a lower dose of AMPH than they received during each of the sensitization sessions (i.e. injections 1-7). Therefore, group differences were determined for the sensitization sessions separately from the challenge test.

Sensitization sessions (injections 1-7). Following the first injection of AMPH, the overall stereotypy scores for female-2.6 animals were greater than for males $(U=3, P<0.001$; Fig. 1A), but repetitive sniffing scores were not $(U=27, P=0.15$;

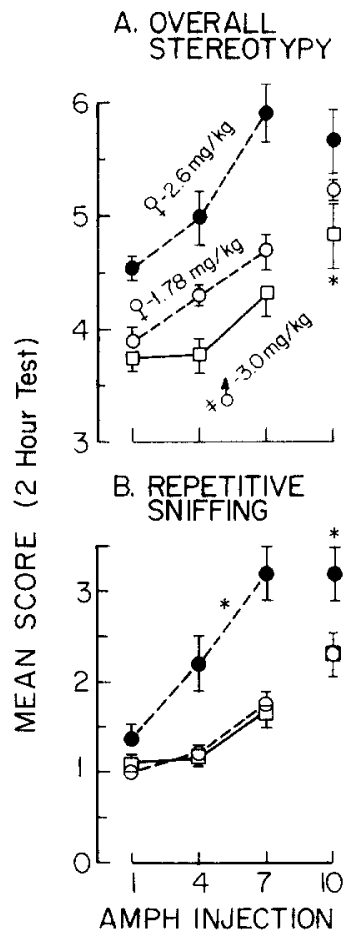

Fig. 1. The effects of repeated amphetamine (AMPH) treatment on overall stereotypy and stereotyped sniffing behavior in male and female rats. Male animals $(n=11)$ received $3.0 \mathrm{mg} / \mathrm{kg} \mathrm{AMPH}$, and female rats either 2.6 or $1.78 \mathrm{mg} / \mathrm{kg}$ ('female-2.6' $[n=8]$ and 'female-1.78' $[n=9]$ animals, respectively; see text). All females received a challenge injection of $1.78 \mathrm{mg} / \mathrm{kg}$ AMPH on the tenth day of AMPH treatment, whereas males again received $3.0 \mathrm{mg} / \mathrm{kg}$. The points represent the average ( \pm S.E.M.) stereotypy scores during the $2 \mathrm{~h}$ following AMPH administration. Separate statistical analyses were conducted on the scores for the 'sensitization' test sessions (injections 1-7), and the 'challenge' session (tenth injection; see text). The symbols represent statistical differences between the indicated group and the other two groups: ${ }^{\ddagger} P=0.059,{ }^{*} P<0.05$ (see text for details).

Fig. 1B). In contrast. there was no difference in either overall stereotypy or stereotyped sniffing between female-1.78 and male animals following the 1st day of AMPH treatment (i.e. 1st test session; overall stereotypy: $P=0.18$; repetitive sniffing: $P>0.5$, based on the median test).

Fig. $1 \mathrm{~A}$ shows there was a progressive enhancement in overall stereotypy scores with successive injections in both males and females (Friedman's analyses of variance conducted on the average scores for the 2 -h test sessions were 
significant for all groups: males, $\chi^{2}=5.9$, $P=0.05$; female- $1.78, \chi^{2}=14.2, P<0.001$; female-2.6, $\chi^{2}=9.2, P=0.01$ ). Analysis of the time course of behavior over each test session gave the same result. To determine if there were sex differences in the rate of sensitization the stereotypy ratings for male and female rats were compared using profile analyses. The profile for male rats differed from the profile of both the female-1.78 and female-2.6 groups at the $P=0.059$ level of significance (i.e. in Fig. 1A the curves are not parallel; $F_{2,17}=3.4, P=0.059$; $F_{2,16}=3.4, P=0.059$, respectively). This interaction was largely accounted for by the difference in scores between the first and second test sessions (i.e. injections 1-4), which was much greater for females than for males (Fig. 1A). A profile analysis comparing the curves of the two female groups resulted in a main effect of dose ( $F=21.7, P<0.001$ ), but no interaction (i.e. the curves are parallel; $F=2.4, P=0.12$ ).

Fig. 1B illustrates the stereotyped sniffing scores were also progressively enhanced in both male and female rats (Friedman's ANOVA's: males, $\chi^{2}=10.4, \quad P=0.005 ; \quad$ female-1.78, $\chi^{2}=12.1, \quad P=0.002 ;$ female- $2.6, \quad \chi^{2}=11.4$, $P=0.003$ ). Consistent with the differences in overall stereotyped behavior, there was a sex difference in the magnitude of the enhancement in stereotyped sniffing over test sessions 1-7. The female-2.6 group showed a greater enhancement in stereotyped sniffing than males (profile analysis interaction, $F=6.6, P=0.008$ ). Animals in the female-2.6 group also showed a greater rate of sensitization than those in the female-1.78 group $(F=5.6, P=0.016)$.

Challenge test. A further conservative test for sex differences in sensitization was conducted on the 10th injection day, when all female animals received a challenge with $1.78 \mathrm{mg} / \mathrm{kg} \mathrm{AMPH}$, and males again received $3.0 \mathrm{mg} / \mathrm{kg}$. These doses did not distinguish the overall stereotypy ratings or repetitive sniffing ratings of male and female rats following the first injection of AMPH (Fig. 1). After repeated AMPH treatment, however, a $1.78 \mathrm{mg} / \mathrm{kg}$ challenge produced greater stereotyped behavior in females than did a $3.0 \mathrm{mg} / \mathrm{kg}$ challenge in males (Fig. 1). Female-2.6 animals given $1.78 \mathrm{mg} / \mathrm{kg}$ showed both greater overall stereotypy ( $P=0.015$, based on the median test) and stereotyped sniffing behavior than did males given $3.0 \mathrm{mg} / \mathrm{kg}(U=17, P=0.024)$. The female1.78 group showed greater overall stereotypy during the challenge test than did males ( $P=0.025$, based on the median test), but did not differ from males in stereotyped sniffing behavior $(U=43)$.

It is interesting that the two female groups also differed from each other during the challenge test, even though both received the same dose of AMPH. Female-1.78 rats had significantly lower scores for repetitive sniffing ( $U=11.5$, $P=0.018$ ), and also a non-significant trend for lower overall stereotypy scores (median test $P=0.088$ ) than female- 2.6 animals. This suggests that pretreatment with the higher dose of AMPHresulted in greater sensitization.

Saline test. All animals received an injection of saline 3-4 days after the last AMPH treatment. The average ( \pm S.E.M.) overall stereotypy scores during the 1 -h test session were $3.22( \pm 0.10)$ in the female-1.78 group, $3.06( \pm 0.15)$ in the female2.6 group, and $2.36( \pm 0.10)$ in males. These scores were significantly lower than the average 1-h scores produced by the initial AMPH injection $\quad(4.11 \pm 0.16, \quad 4.62 \pm 0.12, \quad$ and $3.98 \pm 0.14 ; P=0.007, P=0.01$, and $P=0.003$; Wilcoxon signed-rank tests). The average scores for repetitive sniffing behavior were also significantly lower during the saline test session than during the first AMPH test session ( $P$ 's $<0.02$ ). The two female groups did not differ in their response to an injection of saline ( $P$ 's $>0.5$ ).

\section{Changes in locomotor activity following repeated AMPH administration}

Habituation. In addition to rating stereotyped behavior, an automated method was used to continuously record locomotor activity. Female-1.78 and female-2.6 animals did not differ in the total distance traveled during habituation (data not shown), and therefore these data were pooled for statistically comparing males and females. Females showed greater spontaneous locomotor activity during habituation than males (two-way ANOVA with repeated measures on one factor - 
influence of sex, $F=27.2, P<0.001$ ), but the distance traveled during habituation did not change with repeated testing in either male or female rats $(F$ 's $<1.0)$.

Amphetamine. Fig. 2 shows the effects of repeated AMPH treatment on the time course of locomotor activity in each of the 3 groups. There was a significant change in the pattern of locomotor activity in both female groups by the 4th injection of AMPH (Figs. 2B and 3C; $P$ 's $<0.01$ ), but the pattern of change was very different in the female-1.78 group than in the female-2.6 group. During the first AMPH test, females in both groups showed enhanced locomotion as the predominant response. During subsequent tests the female-1.78 group exhibited a

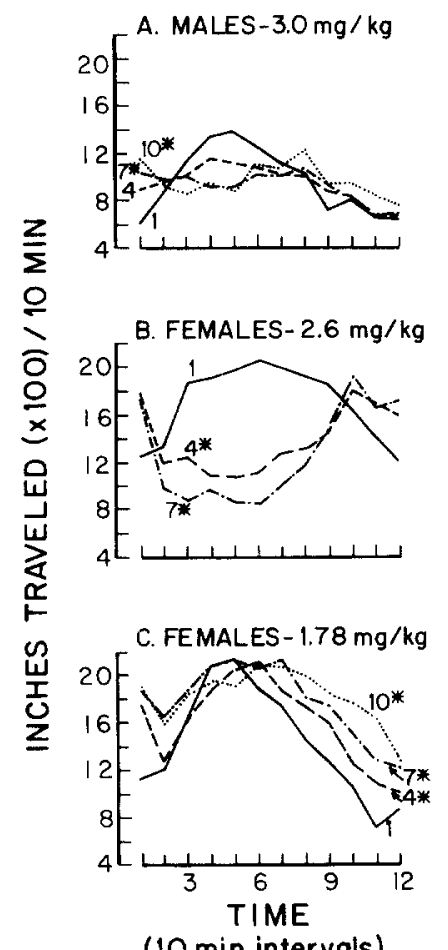

(10 min intervals)

Fig. 2. The effects of repeated amphetamine (AMPH) treatment on the time course of locomotor activity (distance traveled) during 2-h test sessions, in the male and female rats described in Fig. 1. The mean number of inches traveled over each 10-min interval following the 1st, 4th, 7th and 10th injections of AMPH are shown. The asterisks indicate test sessions that differ significantly from the first test session. Note that females were more sensitive to repeated AMPH treatment, showing significant changes in locomotion after only 4 injections, whereas males did not show significant changes until the 7 th injection (see text for details). more rapid onset and longer duration of hyperactivity, but no change in the peak response (Fig. 2C). In contrast, female-2.6 animals showed an initial increase in locomotion, an intermediate phase of reduced locomotion (i.e. a 'stereotypy phase'), and finally, a late second period of increased locomotion (Fig. 2B). In male rats, significant alterations in the pattern of locomotion were not seen until the 7th AMPH treatment (two-way ANOVA's with repeated measures on both factors: 1 st vs 4 th: $F=1.64, P=0.10 ; 1$ st vs 7 th: $F=3.88, P<0.001 ; 1$ st vs 10 th: $F=3.85$, $P<0.001$; all interaction effects). These changes were confined largely to the first hour after AMPH administration, and consisted of a brief initial period of enhanced locomotion followed by a more extended period of slightly decreased locomotion, relative to that following the initial AMPH treatment (Fig. 2).

The changes in locomotor activity in male and female rats were consistent with the stereotypy ratings. In males and the female- 1.78 group, the effect of repeated AMPH treatment on the pattern of locomotion consisted largely of a more rapid onset of hyperactivity; and in the female- 1.78 group by an increase in the duration of the locomotor response. Pretreatment with AMPH did

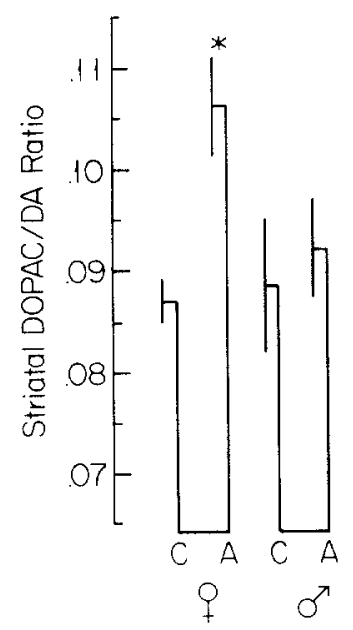

Fig. 3. The mean ( \pm S.E.M.) striatal DOPAC to DA ratios of AMPH-pretreated (A) and control (C) male and female rats ( $n$ 's on Table I). Animals in the female-2.6 and female1.78 groups were pooled (see text). The asterisk indicates that AMPH-pretreatment produced a significant increase in the DOPAC/DA ratio in female $(P=0.02)$, but not male rats. 
not result in the emergence of robust stereotyped behavior in these groups, as indicated by average stereotypy rating of 4-4.5 (normal alert to hyperactivity) and the absence of the multiphasic pattern of locomotion characteristic of the emergence of stereotypy ${ }^{59}$. In contrast, following AMPH pretreatment the female- 2.6 group had an average stereotypy rating near 6 , which is consistent with the decrement in locomotor activity ('stereotypy phase') seen during the peak drug effect $^{59}$.

\section{Neurochemical correlates of behavioral sensitization}

Table I shows the average concentrations of DA, DOPAC, 5-HT and 5-HIAA in the striatum and frontal cortex of AMPH-pretreated animals, and control animals that received no prior treatment. There were no differences in the concentrations of any of these neurochemicals between female-1.78 and female-2.6 rats, or in metabolite/transmitter ratios, and therefore the data for these two groups were pooled for subsequent comparisons. Table I shows that AMPH-pretreatment had no effect on DA or DOPAC concentrations in either brain region, in male or female rats. The concentrations of DOPAC and DA were also expressed as a ratio, because this ratio sometimes provides a sensitive index of DA metabolism $^{4,29,63}$. Fig. 3 illustrates that AMPH pretreatment produced a significant elevation in striatal DOPAC/DA ratios in female $(t=2.09$, $P=0.046)$, but not male rats $(t=0.41)$. There was a non-significant tendency for higher DOPAC/DA ratios in AMPH pretreated females, relative to AMPH-pretreated males $(t=1.84, P=0.078)$. In addition, sensitized females had higher DOPAC/DA ratios in the frontal cortex than control females $(0.359 \pm 0.017$ vs $0.314 \pm 0.013$; mean \pm S.E.M.), but this difference was not statistically significant $(t=1.53$, $P=0.14$ ).

There was an overall sex difference in the concentration of 5-HT in the frontal cortex in control animals (males $>$ females; $t=2.35, P=0.04$; Table I). However, AMPH pretreatment had no effect on 5-HT, 5-HIAA or 5-HIAA/5-HT ratios in any group or structure, except for a small but statistically significant elevation in frontal cortex
5-HIAA concentrations in AMPH-pretreated females $(t=2.01, P=0.05$; Table I).

\section{Sub-group analyses}

In response to a suggestion by $\mathbf{R}$. Kuczenski (personal communication, December $1986^{60}$ ) the male group was subdivided, post hoc, into two subgroups, based on their behavioral response to the tenth injection of AMPH. The animals were ranked according to the cumulative activity scores obtained over the first $60 \mathrm{~min}$ after AMPH administration, and then subdivided into those having relatively high activity scores $(>6,250$ inches) and those having relatively low activity scores ( $<6,250$ inches). These groups will be referred to as the HI and LO activity groups, respectively, and of course they differ significantly from one another because this division resulted in no overlap in their activity scores $(t=4.1$, $P=0.003$ ). It is suggested that the LO activity animals showed greater behavioral sensitization than $\mathrm{HI}$ activity animals for the following reasons. (1) The HI and LO subgroups did not differ in their behavioral response to the first injection of AMPH $(t=0.7)$, but came to differ only after repeated AMPH treatment. (2) The HI activity subgroup showed a small $(19 \%)$ but statistically significant elevation in activity between the first and tenth test sessions $(6,337 \pm 612$ vs $7,567 \pm 580$ inches; $t=3.6, P=0.016$, paired $t$-test), whereas the LO activity subgroup showed a $42 \%$ decrease in activity between the first and tenth test sessions $(7,047 \pm 850$ vs $3,935 \pm 743$ inches; paired $t=3.1, P=0.036$ ). This latter pattern is characteristic of highly sensitized animals, and indeed was the pattern of change seen in female-2.6 rats (illustrated in Fig. 2). In addition, the reduced activity seen in LO activity males during the tenth test session was associated with vigorous stereotyped behavior (scores of $6-8$ ). Therefore, by dividing the group in this manner it is probable that the most sensitized animals were placed in one subgroup and the least sensitized in the other. Only after the subgroups were established by consideration of the behavioral data were the neurochemical data reanalyzed. (It should be kept in mind that the neurochemical data were obtained over a week 


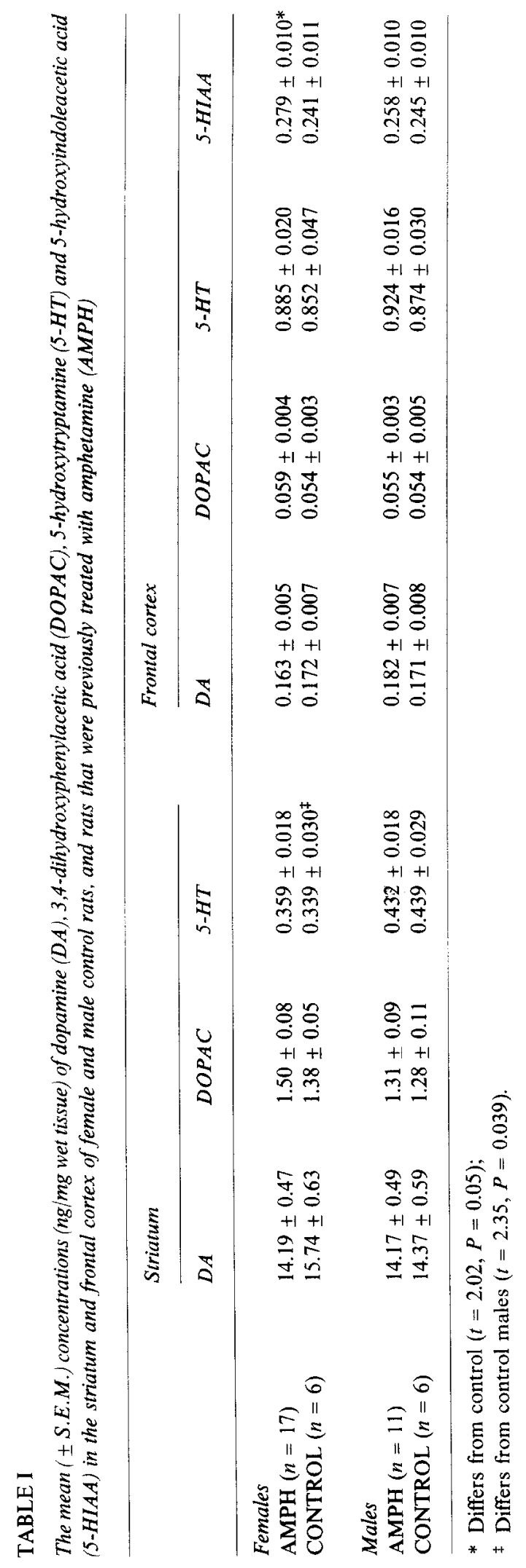




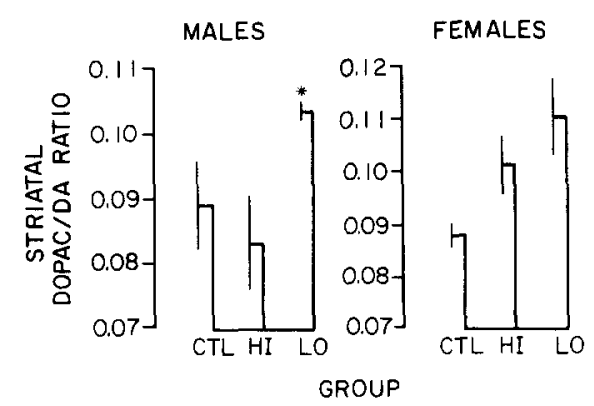

Fig. 4. The mean ( \pm S.E.M.) striatal DOPAC/DA ratios in control (CTL) and AMPH-pretreated male (left) and female (right) rats. The AMPH pretreated groups were subdivided into high activity (HI) and low activity (LO) subgroups on the basis of their locomotor response to the last injection of AMPH, which was given over a week earlier (see text). The asterisk indicates that the AMPH-treated male animals fell into two neurochemically distinct subgroups $(P=0.027)$, but the females did not. (Group $n$ 's from left to right on the figure: $6,6,5,6,8,9$.)

after the last injection of AMPH, and when the animals were in a non-drugged state.)

Fig. 4 (left) shows the mean striatal DOPAC/ DA ratios for high (HI) and low activity (LO) males. LO males had significantly higher DOPAC/DA ratios than HI males $(t=2.21$, $P=0.05$ ). The HI activity males did not differ from control males, whereas there was a tendency for elevated striatal DOPAC/DA ratios in LO activity males, relative to controls (Fig. 4, left; $t=1.79, \quad P=0.053, \quad 1$-tail). There were no subgroup differences for any other neurochemical measure. The same analysis of females did not reveal any differences between $\mathrm{HI}$ versus $\mathrm{LO}$ activity animals for any neurochemical measure (e.g. Fig. 4, right).

\section{DISCUSSION}

The major question addressed here was whether there are sex differences in the sensitization of stereotyped behavior or locomotion produced by repeated AMPH treatment. The answer is yes. Repeated AMPH treatment produced a greater enhancement in overall stereotypy scores and ratings of repetitive sniffing in female than in male rats. Repeated AMPH treatment also produced more robust changes in automated locomotor activity counts in female than in male rats.
Sex differences in the sensitization produced by either AMPH or cocaine have now been reported in 6 different studies, using 5 different injection regimens $^{7,14,40,44,50}$ (also, present study). Taken together, the available studies provide strong evidence for sex differences in the sensitization produced by stimulant drugs, and establish that the effect is not peculiar to just one AMPH-induced behavior.

It is important to consider whether sex differences in behavioral sensitization are due to pharmacokinetic factors. Male rats metabolize AMPH more rapidly than females, primarily due to the influence of androgens on liver microsomal enzymes, and therefore, if males and females are given the same systemic dose of AMPH, brain levels are higher in females ${ }^{3,10,22,34}$. Of course, if male rats received a lower dose of AMPH than females, in terms of the amount of AMPH reaching the brain, this could account for the lower rate of sensitization in males. However, a sex difference in AMPH metabolism is probably not responsible for the sex difference in behavioral sensitization $^{6}$, for the following reasons.

In all the studies showing a sex difference in sensitization to AMPH, male rats received a higher systemic dose of AMPH than females to compensate for the sex difference in AMPH metabolism $^{7,44,50}$ (also, present study). The systemic doses used to produce equivalent brain levels of AMPH in male and female rats were based on extensive experiments in which brain and striatal levels of AMPH were measured after systemic treatment with a wide range of doses $(1-10 \mathrm{mg} / \mathrm{kg})$, and in the same strain of rat as used in the behavioral experiments ${ }^{3}$. Therefore, the sex difference in sensitization persists when males and females are treated with doses of AMPH known to produce comparable brain concentrations of the drug. In addition, female rats given a dose of AMPH that was estimated would produce brain levels of AMPH 33\% lower than in males also showed more robust sensitization than males. The female-1.78 group showed a greater enhancement in overall stereotypy ratings than males $(P=0.059)$, and more robust changes in automated locomotor activity counts. Furthermore, both female groups showed significant 
changes in behavior by the fourth AMPH test, but males did not show significant changes in behavior until after the seventh test, suggesting that females sensitized more rapidly than males.

Nevertheless, even after controlling for pharmacokinetic variables to insure that males and females receive equivalent brain levels of AMPH, females frequently show a greater acute behavioral response to the drug than do males. This is clearly illustrated in Fig. 1 of Becker et al. ${ }^{3}$, which shows striking sex differences and estrous cycle-related differences in AMPH-induced rotational behavior in the absence of differences in the brain levels of AMPH. This sex difference often persists even if females are given a dose of AMPH that produces lower brain levels of AMPH than in a comparison group of males (present study and refs. 3,5,44,56). Therefore, controlling for the sex difference in AMPH metabolism by titrating doses, as in the present study, does not 'control' for constitutional differences in the response of males and females to equivalent brain levels of AMPH. But it is not meant to. In fact, it is probably impossible to completely compensate for the sex difference in the response to AMPH, because this sex difference is not just due to sex differences in AMPH metabolism, but to qualitative differences in the response of males and females to AMPH.

That sex differences in the acute and chronic effects of AMPH are not just due to pharmacodynamic factors is further supported by reports that females are more responsive than males to a wide range of activating stimuli ${ }^{21,25,31,54}$, and that females show more robust sensitization to repeated stress than do males. For example, females show a greater sensitization of the corticosterone response induced by repeated footshock stress than do males ${ }^{17}$, and prior exposure to restraint stress results in a greater enhancement in AMPHinduced stereotyped behavior in females than in males ${ }^{7}$. Obviously, sex differences in the sensitization produced by stress cannot be due to pharmacodynamic factors. Therefore, to the extent that the sensitization produced by stimulants and stress represent a common phenomenon, as suggested by many reports ${ }^{1,2,18,32,46,51}$, sex differences in sensitization are not attributable to pharmacodynamic factors.
This seemingly general sex difference in responsivity to activating stimuli, including AMPH, raises the question whether variation in the susceptibility to sensitization due to sex is due to sex differences in the propensity for change (sensitization), or whether it is a reflection of the responsiveness to an acute injection of AMPH. Stated another way, are the constitutional differences responsible for individual variation in the acute response to AMPH also responsible for individual variation in the susceptibility to sensitization? If so, the response to the first injection of AMPH should predict the susceptibility to sensitization. This was not the case in the present study, because highly sensitized animals did not differ from less sensitized animals following the first injection of AMPH. Robinson ${ }^{44}$ reached a similar conclusion in a study on the sensitization of AMPH-induced rotational behavior. Perhaps most convincing are studies by Shuster et al ${ }^{58}$ on the sensitization produced by repeated cocaine treatment in inbred strains of mice. These researchers dissociated the acute response to cocaine and the susceptibility to sensitization, concluding that, "the initial response to cocaine and the development of sensitization are controlled by different genetic determinants" (p. 185). Therefore, the sex difference in sensitization is probably not simply an extension of a sex difference in the initial responsiveness to AMPH (see ref. 45 for a more complete discussion of this point).

Most of the behaviors sensitized by AMPH are largely due to AMPH-stimulated DA release ${ }^{11,19}$, and it has been suggested that sensitization is due to enduring changes in mesotelencephalic DA systems (ref. 48 for review). In the present study AMPH pretreatment had no effect on 'resting' DA or DOPAC concentrations in the striatum or frontal cortex, but AMPH-pretreated female rats did have significantly higher DOPAC/ DA ratios than non-treated control animals. In some experimental situations an increase in metabolite/transmitter ratios reflects enhanced neurotransmitter utilization ${ }^{4,29,63}$, but unfortunately, changes in metabolism are not always a reliable indicator of utilization ${ }^{9,67}$. The change in DOPAC/DA ratios found here is particularly difficult to interpret because it was not ac- 
companied by a significant change in metabolite concentrations, as is usually the case if utilization is enhanced ${ }^{27,53}$. Nevertheless, the elevation in DOPAC/DA ratios seen in AMPH-pretreated female rats may reflect a sensitization-related enhancement in striatal DA metabolism, for the following reasons.

First, the results were replicated in an independent experiment using a similar injection regimen, and most importantly, the elevation in metabolite/transmitter ratios was accompanied by a significant elevation in striatal homovanillic acid concentrations in this experiment ${ }^{7}$. Similarly, Robinson and Camp ${ }^{52}$ found that DOPAC/DA ratios and DOPAC concentrations were elevated in the striatum and nucleus accumbens of female rats given a regimen of escalating doses of AMPH, and Watanabe ${ }^{66}$ reported a similar observation in male rats. In a related experiment, Schuster et al. ${ }^{57}$ found that methylphenidate pretreatment in mice enhanced striatal DA turnover, as indicated by the rate of decline in DA after tyrosine hydroxylase inhibition, and this was accompanied by increased DOPAC/DA ratios but no change in DA or DOPAC concentrations. In summary, sensitization-related increases in striatal DOPAC/DA ratios are typically associated with an increase in striatal DA metabolism, supporting the argument that the elevated DOPAC/DA ratios reported here do reflect a change in striatal DA metabolism. Metabolite/transmitter ratios are probably more sensitive indicators of changes in metabolism than metabolite concentrations alone, and this is why just the ratio was elevated in this particular study. To the extent that an increase in striatal DA metabolism is indicative of increased DA utilization, the present results are consistent with the idea that AMPH pretreatment enhances striatal DA utilization in female animals. This idea is further supported by reports that in female rats, prior exposure to AMPH enhances AMPH-, potassiumand electrical stimulation-induced striatal DA release measured in vitro ${ }^{8,47,68}$.

In female rats sensitization to AMPH is consistently accompanied by both an increase in striatal DA metabolism (present study and refs. 7,52), and in striatal DA release in vitro ${ }^{8,50,68}$. In male rats, however, the picture is not as clear. AMPHpretreated male rats frequently do not show enduring changes in basal striatal DA metabolism or utilization ${ }^{7,28,36,38}$ (cf. ref. 66). If changes in striatal metabolism are related to the development of behavioral sensitization, why do both males and females show behavioral sensitization but only females show increases in striatal DA metabolism? There are a number of possible answers to this question. One is that changes in striatal DA activity are not causally related to behavioral sensitization. But like females, male rats do show a sensitization-related increase in striatal DA utilization/release when measures are made following an additional AMPH 'challenge'26, $28,38,47$. Perhaps measures of basal DA activity do not provide a sensitive index of the neural changes produced by repeated AMPH treatment, and the change is primarily characterized by an enhanced response to a subsequent challenge ${ }^{48}$. Changes in basal striatal DA activity may be more apparent in females because the sex difference in behavioral sensitization is accompanied by a sex difference in the magnitude of the neurochemical consequences of AMPH treatment. It is also possible that the kinds of neurochemical measures reported here do not provide a true reflection of basal conditions, but a rapid and exaggerated response to stress ${ }^{1}$. This would be expected to be more pronounced in females ${ }^{21,25,45}$. The idea that changes in striatal DA metabolism are related to the development of behavioral sensitization is further supported by analysis of the high and low activity subgroups. The presumably more highly sensitized males (LO activity) had significantly greater DOPAC/DA ratios than $\mathrm{HI}$ activity males. The difference between the two subgroups was only marginally significant, but is reliable, because the effect has been replicated ${ }^{7}$.

It is particularly interesting that the male group was divisible into two neurochemically distinguishable subgroups and the female group was not. This finding has also been replicated ${ }^{7}$, and it suggests that male populations may be more heterogeneous in their response to repeated AMPH treatment than female populations. This greater heterogeneity could make it relatively difficult to identify neurochemical correlates of sensi- 
tization in male rats ${ }^{48}$. Many factors may contribute to heterogeneity in male populations. For example, it was previously suggested that a testicular hormone attenuates the development of sensitization $^{44,50}$, and therefore it is possible that HI activity males have higher levels of circulating androgens than LO activity males. Evidence to support the idea that males constitute a more variable or heterogeneous population than females for many biological traits was recently reviewed by Juraska ${ }^{20}$.

In summary, the results reported here, in combination with other studies, suggest that: (1) the repeated administration of psychomotor stimulant drugs produces greater behavioral sensitization in female than in male rats ${ }^{7,14.44,50}$; (2) neural correlates of sensitization may be more readily identifiable in female than in male rats; and ( 3 ) as a group, males may be more heterogeneous than females in their susceptibility to sensitization. It is interesting that there are similar sex differences in the sensitization produced by repeated intermittent stress ${ }^{7,17}$. The available evidence suggests there is a general sex difference in the responsiveness of brain DA systems to repetitive activation, and that females are more susceptible to the sensitization produced by repeated exposure to activating stimuli than males. The neurobiological basis and adaptive significance of such a sex difference is unknown.

\section{ACKNOWLEDGEMENTS}

This research was submitted by D.M.C. in partial fulfillment of requirements for a Ph.D. in Psychology (Psychobiology) at The University of Michigan, Ann Arbor. A preliminary version was reported earlier: Soc. Neurosci. Abstr., 11 (1985) 550. The research was supported by grants from the NIH (MH37277), Scottish Rite Schizophrenia Research Program and Rackham School of Graduate Studies. T.E.R. was supported by a Research Career Development Award (NS00844). We thank Dr. J.B. Becker for her comments and advice during all stages of the project.

\section{REFERENCES}

1 Antelman, S.M. and Chiodo, L.A., Amphetamine as a stressor. In I. Creese (Ed.), Stimulants: Neurochemical. Behavioral, and Clinical Perspectives, Raven, New York, 1983, pp. 269-299.

2 Antelman, S.M., Eichler, A.J., Black, C.A. and Kocan, D., Interchangeability of stress and amphetamine in sensitization, Science, 207 (1980) 329-331.

3 Becker, J.B., Robinson, T.E. and Lorenz, K.A., Sex differences and estrous cycle variations in amphetamineelicited rotational behavior, Eur. J. Pharmacol., 80 (1982) 65-72.

4 Blanc, G., Hervé, D., Simon, H., Lisoprawski, A., Glowinski, J. and Tassin, J.P., Response to stress of mesocortico-frontal dopaminergic neurones in rats after long-term isolation, Nature (Lond.), 284 (1980) 265-267.

5 Brass, C.A. and Glick, S.D., Sex differences in druginduced rotation in two strains of rats, Brain Res., 223 (1981) 229-234.

6 Browne, R.G. and Segal, D.S., Metabolic and experiential factors in the behavioral response to repeated amphetamine, Pharmacol. Biochem. Behav., 6 (1977) 545-552.

7 Camp, D.M. and Robinson, T.E., Susceptibility to sensitization. II. The influence of gonadal hormones on enduring changes in brain monoamines and behavior produced by the repeated administration of amphetamine or restraint stress, Behav. Brain Res., 30 (1988) 69-88.

8 Castañeda, E., Becker, J.B. and Robinson, T.E., Depolarization-induced striatal dopamine release in rats sensitized to amphetamine, Soc. Neurosci. Abstr., 12 (1986) 1141.

9 Commissiong, J.W., Monoamine metabolites: their relationship and lack of relationship to monoaminergic neuronal activity. Biochem. Pharmacol, 34 (1985) 1127-1131.

10 Conney, P.H., Pharmacological implications of microsomal enzyme induction, Pharmacol. Rev., 19 (1967) 317-366.

11 Costall, B. and Naylor, R.J., Mesolimbic and extrapyramidal sites for the mediation of stereotyped behavior patterns and hyperactivity by amphetamine and apomorphine in the rat. In E.H. Ellinwood and M.M. Kilbey (Eds.), Cocaine and Other Stimulants, Plenum, New York, 1977, pp. 47-76.

12 Echols, S.D., Circling of mice bearing unilateral striatal lesions: development of increased response to $D$-amphetamine, Life Sci, 21 (1977) 563-568.

13 Ellinwood, Jr., E.H. and Balster, R.L., Rating the behavioral effects of amphetamine, Eur. J. Pharmacol., 28 (1974) 35-41.

14 Glick, S.D. and Hinds, P.A., Sex differences in sensitization to cocaine-induced rotation, Eur.J. Pharmacol., 99 (1984) 119-121.

15 Glick, S.D., Shapiro, R.M., Drew, K.L., Hinds, P.A. and Carlson, J.N., Differences in spontaneous and amphe- 
tamine-induced rotational behavior, and in sensitization to amphetamine, among Sprague-Dawley derived rats from different sources, Physiol. Behav., 38 (1986) 67-70.

16 Heffner, T.G., Hartman, J.A. and Seiden, L.S., A rapid method for the regional dissection of the rat brain, Pharmacol. Biochem. Behav., 13 (1980) 453-456.

17 Hennessy, J.W., Levin, R. and Levine, S., Influence of experiental factors and gonadal hormones on pituitary-adrenal response of the mouse to novelty and electric shock, J. Comp. Physiol. Physiol., 91 (1977) 770-777.

18 Herman, J.P., Stinus, L. and Le Moal, M., Repeated stress increases locomotor response to amphetamine, Psychopharmacology, 84 (1984) 431-435.

19 Iversen, S.D., Brain dopamine systems and behavior. In L.L. Iversen, S.D. Iversen and S.H. Snyder (Eds.), Handbook of Psychopharmacology, Vol. 8, Plenum, New York, 1977, pp. 333-383.

20 Juraska, J.M., Sex differences in developmental plasticity of behavior and the brain. In W.T. Greenough and J.M. Juraska (Eds.), Developmental Neuropsychobiology, Academic, New York, 1986, pp. 409-422.

21 Kant, G.J., Lenox, R.H., Bunnell, B.N., Mougey, E.H., Pennington, L.L. and Meyerhoff, J.L., Comparison of stress response in male and female rats: pituitary cyclic AMP and plasma prolactin, growth hormone and corticosterone, Psychoneuroendocrinology, 8 (1986) 421-418.

22 Kato, R., Sex-related differences in drug metabolism, Drug Metabol. Rev., 3 (1974) 1-32.

23 Kelly, P.H., Drug-induced motor behavior. In L.L. Iversen, S.D. Iversen and S.H. Snyder (Eds.), Handbook of Psychopharmacology, Vol. 8, Plenum, New York, 1977, pp. 295-331.

24 Klawans, H.L. and Margolin, D.I., Amphetamineinduced dopaminergic hypersensitivity in guinea pigs, Arch. Gen. Psychiatry, 32 (1975) 725-732.

25 Kitay, J.I., Sex differences in adrenal cortical secretion in the rat, Endocrinology, 68 (1961) 818-824.

26 Kolta, M.B., Shreve, P., De Souza, V. and Uretsky, N.J., Time course of the development of the enhanced behavioral and biochemical responses to amphetamine after pretreatment with amphetamine, Neuropharmacology, 24 (1985) 823-829.

27 Korf, J., Grasdijk, L. and Westerink, B.H.C., Effects of electrical stimulation of the nigrostriatal pathway of the rat on dopamine metabolism, J. Neurochem., 26 (1976) 579-584.

28 Kuczenski, R. and Leith, N.J., Chronic amphetamine: is dopamine a link in or mediator of the development of tolerance and reverse tolerance?, Pharmacol. Biochem. Behav., 15 (1981) 405-413.

29 Lavielle, S., Tassin, J.P., Thierry, A.M., Blanc, G., Herve, D., Barthelemy, C. and Glowinski, J., Blockade by benzodiazepines of the selective high increase in dopamine turnover induced by stress in mesocortical dopaminergic neurons of the rat. Brain Res., 168 (1978) 585-594.

30 Leith, N.J. and Kuczenski, R., Two dissociable components of behavioral sensitization following repeated amphetamine administration, Psychopharmacology, 76 (1982) 310-315.

31 Livezey, G.T., Miller, J.M. and Vogel, W.H., Plasma norepinephrine and corticosterone stress responses to restraint in individual male and female rats, and their correlations, Neurosci. Lett., 62 (1985) 51-56.

32 MacLennan, A.J. and Maier, S.F., Coping and the stress-induced potentiation of stimulant stereotypy in the rat, Science, 219 (1983) 1091-1093.

33 Magos, L., Persistence of the effect of amphetamine on stereotyped activity in rats, Eur. J. Pharmacol., 6 (1969) 200-201.

34 Meyer, E.M., Jr. and Lytle, L.D., Sex-related differences in the physiological disposition of amphetamine and its metabolites in the rat, Proc. West. Pharmacol. Soc., 21 (1978) 313-316.

35 Michigan Statistical Research Laboratory Staff, Elementary Statistics Using MIDAS, 2nd edn., The University of Michigan Statistical Research Laboratory, Ann Arbor, Michigan, 1976.

36 Mittleman, G., Castañeda, E., Robinson, T.E. and Valenstein, E.S., The propensity for non-regulatory ingestive behavior is related to differences in dopamine systems: behavioral and biochemical evidence, Behav. Neurosci., 100 (1986) 213-220.

37 Morrison, D.F., Multivariate Statistical Methods, McGraw-Hill, New York, 1967.

38 Nishikawa, T., Mataga, N., Takashima, M. and Toru, M., Behavioral sensitization and relative hyperresponsiveness of striatal and limbic dopaminergic neurons after repeated methamphetamine treatment, Eur. J. Pharmacol., 88 (1983) 195-203.

39 Pickens, R. and Dougherty, J.A., Conditioning of the activity effects of drugs. In T.I. Thompson and R. Pickens (Eds.), Stimulus Properties of Drugs, Appleton-CenturyCrofts, New York, 1971, pp. 39-50.

40 Post, R.M. and Contel, N.R., Human and animal studies of cocaine: implications for the development of behavioral pathology. In I. Creese (Ed.), Stimulants: Neurochemical, Behavioral and Clinical Perspectives, Raven, New York, 1983, pp. 169-203.

41 Post, R.M., Lockfield, A., Squillace, K.M. and Contel, N.R., Drug-environment interaction; context dependency of cocaine-induced behavioral sensitization, Life Sci., 28 (1981) 755-760.

42 Ranje, C. and Ungerstedt, U., Chronic amphetamine treatment: vast individual differences in performing a learned response, Eur. J. Pharmacol, 29 (1974) 307-311.

43 Rebec, G.V. and Segal, D.S., Apparent tolerance to some aspects of amphetamine stereotypy with long-term treatment, Pharmacol. Biochem. Behav., 13 (1980) 793-797.

44 Robinson, T.E., Behavioral sensitization: characterization of enduring changes in rotational behavior produced by intermittent injections of amphetamine in male and female rats, Psychopharmacology, 84 (1984) 466-475.

45 Robinson, T.E., Stimulant drugs and stress: factors influencing the susceptibility to sensitization. In $\mathrm{C}$. 
Barnes and P. Kalivas (Eds.), Sensitization of the Nervous System, Telford, Caldwell, NJ, in press.

46 Robinson, T.E., Angus, A.L. and Becker, J.B., Sensitization to stress: the enduring effects of prior stress on amphetamine-induced rotational behavior, Life $S c i ., 37$ (1985) 1039-1042.

47 Robinson, T.E. and Becker, J.B., Behavioral sensitization is accompanied by an enhancement in amphetaminestimulated dopamine release from striatal tissue in vitro, Eur. J. Pharmacol, 85 (1982) 253-254.

48 Robinson, T.E. and Becker, J.B., Enduring changes in brain and behavior produced by chronic amphetamine administration: a review and evaluation of animal models of amphetamine psychosis, Brain Res. Rev., 11 (1986) 157-198.

49 Robinson, T.E., Becker, J.B., Moore, C.J., Castañeda, E. and Mittleman, G., Enduring enhancement in frontal cortex dopamine utilization in an animal model of amphetamine psychosis, Brain Res., 343 (1985) 374-377.

50 Robinson, T.E., Becker, J.B. and Presty, S.K., Long-term facilitation of amphetamine-induced rotational behavior and striatal dopamine release produced by a single exposure to amphetamine: sex differences, Brain Res., 253 (1982) 231-241.

51 Robinson, T.E., Becker, J.B., Young, E.A., Akil, H. and Castañeda, E., The effects of footshock stress on regional brain dopamine metabolism and pituitary $\beta$-endorphin release in rats previously sensitized to amphetamine, Neuropharmacology, 26 (1987) 679-691.

52 Robinson, T.E. and Camp, D.M., Long-lasting effects of escalating doses of $\mathrm{D}$-amphetamine on brain monoamines, amphetamine-induced stereotyped behavior and spontaneous nocturnal locomotion, Pharmacol. Biochem. Behav., 26 (1987) 821-827.

53 Roth, R.H., Murrin, C.L. and Walters, J.R., Central dopaminergic neurons: effects of alterations in impulse flow on the accumulation of dihydroxyphenylacetic acid, Eur. J. Pharmacol., 36 (1976) 163-171.

54 Sassenrath, E.N., Increased adrenal responsiveness related to social stress in rhesus monkeys, Horm. Behav., 1 (1970) 283-298.

55 Schiff, S.R., Conditioned dopaminergic activity, Biol. Psychiatry, 17 (1982) 135-154.

56 Schneider, B.F. and Norton, S., Circadian and sex differences in hyperactivity produced by amphetamine in rats, Physiol. Behav., 22 (1979) 47-51.

57 Schuster, L., Hudson, J., Anton, M. and Righi, D., Sensitization of mice to methylphenidate, Psychopharmacology, 77 (1982) 31-36.
58 Schuster, L., Yu, G. and Bates, A., Sensitization to cocaine stimulation in mice, Psychopharmacology, 52 (1977) 185-190.

59 Segal, D.S., Behavioral and neurochemical correlates of repeated D-amphetamine administration. In A.J. Mandell (Ed.), Neurobiological Mechanisms of Adaptation and Behavior, Raven, New York, 1975, pp. 247-262.

60 Segal, D.S. and Kuczenski, R., Individual differences in responsiveness to single and repeated amphetamine administration: behavioral characteristics and neurochemical correlates, $J$. Pharmacol. exp. Ther., 242 (1987) 917-926.

61 Segal, D.S. and Mandell, A.J., Long-term administration of D-amphetamine: progressive augmentation of motor activity and stereotypy, Pharmacol. Biochem. Behav., 2 (1974) 249-255.

62 Stewart, J. and Vezina, P., Environment-specific enhancement of the hyperactivity induced by systemic or intra-VTA morphine injections in rats pre-exposed to amphetamine, Psychobiology, 15 (1987) 144-153.

63 Szentendrei, T., Herman, J.P., Kanyicska, B. and Fekete, M.I.K., Stress-induced changes in dopamine metabolism of rat brain cortex as well as plasma corticosterone and prolactin levels - effects of diazepam and tofisopam. In E. Usdin, R. Kvetnansky and I. Kopin (Eds.), Catecholamines and Stress: Recent Advances, Elsevier, Amsterdam, 1980, pp. 161-166.

64 Tilson, H.A. and Rech, R.H., Conditioned drug effects and absence of tolerance to D-amphetamine-induced motor activity, Pharmacol. Biochem. Behav., 1 (1973) 149-153.

65 Ungerstedt, U., Central dopamine mechanisms and unconditioned behavior. In A.S. Horn, J. Korf and B.H. Westerink (Eds.), The Neurobiology of Dopamine, Academic, London, 1979, pp. 577-596.

66 Watanabe, H., Subchronic methamphetamine treatment selectively attenuates apomorphine-induced decrease in 3,4-dihydroxyphenylacetic acid level in mesolimbic dopaminergic regions, Life Sci., 37 (1985) 2319-2325.

67 Westerink, B.H.C., Sequence and significance of dopamine metabolism in the rat brain, Neurochem. Int., 7 (1985) 221-227.

68 Wilcox, R.A., Robinson, T.E. and Becker, J.B., Enduring enhancement in amphetamine-stimulated striatal dopamine release in vitro produced by prior exposure to amphetamine or stress in vivo, Eur. J. Pharmacol., 124 (1986) 375-376. 